\title{
Costruzione e validazione del questionario DIDLab "La didattica Laboratoriale: apprendimenti e stili di conduzione con l'approccio IBSE"
}

\section{Construction and validation of the DIDLab questionnaire "Laboratory teaching: IBSE-based learning \& conducting styles"}

\author{
Giuseppa Cappuccio* e Lucia Maniscalco*
}

\begin{abstract}
Riassunto
Il presente lavoro espone gli esiti della costruzione e validazione del questionario "La didattica Laboratoriale: apprendimenti e stili di conduzione con l'approccio IBSE" finalizzato a misurare il grado di conoscenza dell'approccio Inquiry-Based Science Education e lo stile di conduzione nella didattica laboratoriale, che ha visto coinvolti 523 corsisti del corso di specializzazione per le attività di sostegno didattico dell'Università degli Studi di Palermo; il test di misurazione costruito è composto da 42 item suddiviso in cinque macro-aree su scala Likert a 5 punti.

Dall'analisi quantitativa condotta è emerso che il questionario ha buona concordanza, pensato per indagare sulle conoscenze dell'approccio investigativo e migliorare lo stile di conduzione della didattica laboratoriale. Quest'ultima risulta utile a facilitare l'acquisizione di una competenza in ogni ordine di scuola. Il laboratorio, infatti, rappresenta la modalità trasversale che promuove nello studente una preparazione completa e capace di continuo rinnovamento. Dall'analisi sono emersi risultati molto soddisfacenti per quanto riguarda l'attendibilità e la validità del questionario costruito. A seguito dell'analisi fattoriale condotta sono stati estrapolati i due fattori più rappresentativi, denominati Stili di conduzione nella didattica laboratoriale e Conoscenza dell'approccio Inquiry-Based Science Education. Infine, è stata condotta un MANOVA 2 X 2 per esplorare differenze ascrivili agli anni di servizio e al grado di istruzione dove si presta insegnamento. Tale indagine ha rilevato una correlazione tra la conoscenza e l'applicazione dell'approccio IBSE alla didattica laboratoriale
\end{abstract}

\footnotetext{
* Università degli Studi di Palermo.

Excellence and Innovation in Learning and Teaching (ISSNe 2499-507X), 2021, 1
}

Doi: $10.3280 /$ exioa1-2021oa12066 
tanto maggiore quanto più sono gli anni di servizio e all'interno degli ordini di scuola primaria e secondaria di primo grado.

Parole chiave: IBSE, didattica laboratoriale, stili di conduzione, questionario, validazione.

\begin{abstract}
This paper presents the results of the construction and validation of the questionnaire "Laboratory teaching: IBSE-based learning \& conducting styles" aimed to measure the approach Inquiry-Based Science Education knowledge and the conducting style in laboratory teaching, which involved 523 students of the specialization course for the activities of educational support of the University of Palermo; The built-in measuring test consists of 42 items divided into five 5-point Likert macro-areas. The analysis showed that the questionnaire is of good correlation, designed to investigate the knowledge of the investigative approach and improve the style of conducting laboratory teaching. This is useful to facilitate the acquisition of a competence in each school order. The laboratory, in fact, represents the transversal mode which promotes a complete preparation and capable of continuous renewal in the student. The analysis showed very satisfactory results with regard to the reliability and validity of the constructed questionnaire. On the basis of the factorial analysis conducted were extrapolated the two most representative factors, called Styles of conducting in laboratory teaching and Knowledge of the approach InquiryBased Science Education. Finally, a MANOVA 2 X 2 was conducted to explore differences related to the years of service and the level of education where it is taught. This survey found a correlation between the knowledge and the application of the IBSE approach to laboratory teaching as much greater as the years of service and within the primary and secondary schools of first degree.
\end{abstract}

Keywords: IBSE, laboratory education, teaching styles, questionnaire, validation

\title{
1. Introduzione ${ }^{1}$
}

La formazione degli insegnanti in servizio in laboratorio è particolarmente rilevante perché consente di attivare processi di insegnamento/apprendimento in cui gli alunni diventano protagonisti del proprio processo e superano l'atteg-

\footnotetext{
${ }^{1}$ L'articolo, frutto della collaborazione sinergica tra le due ricercatrici, è così suddiviso: G. Cappuccio è autrice dell'Introduzione, dei paragrafi 1 e 2 e delle conclusioni; L. Maniscalco è autrice dei paragrafi 3, 4 e 5 .
} 
giamento di passività e di estraneità che caratterizza spesso il loro atteggiamento di fronte alle lezioni frontali, divenendo in tal modo l'acquisizione di conoscenze momento di apprendimento significativo (Ausubel, 1963; 2004).

Il laboratorio può, quindi, rappresentare la modalità trasversale che caratterizza la didattica e la formazione dei futuri docenti, per promuovere nello studente una preparazione completa e capace di continuo rinnovamento, idonea per la promozione di un apprendimento significativo ovvero in grado di creare nella stessa persona in formazione, consapevolmente ed esplicitamente, un sapere nuovo partendo dai concetti e dai contenuti preesistenti nella sua struttura cognitiva rendendola attraverso l'attivazione nel soggetto, "protagonista" del proprio apprendimento, processi cognitivi attivi in grado di produrre conoscenze stabili e durature (Amenta, 2008; Ausubel, 2004; Pedone, 2012). La didattica laboratoriale diventa in tale prospettiva uno strumento di innovazione nel processo di insegnamento-apprendimento in quanto colui che apprende, illuminato dalla teoria, diventa l'elemento centrale del percorso formativo mettendo in atto quanto appreso dal docente e analizzando, sperimentando e valutando la realtà (Cappuccio \& Maniscalco, 2020). Attraverso la didattica laboratoriale gli studenti hanno la possibilità di confrontarsi, di mettere in atto le abilità critiche, logiche e creative per esplorare la realtà circostante. Tutto questo si coniuga perfettamente con le possibilità che può offrire un laboratorio scientifico Inquiry-Based. Attraverso l'approccio Inquiry si attua un processo di investigazione in cui colui che apprende: simula una ricerca scientifica, fa osservazioni, si pone delle domande, cerca le risposte consultando, esegue esperimenti, raccoglie dati, li analizza e propone soluzioni (Uzezi Gladys \& Zainab, 2017).

In linea con gli studi sull'Inquiry Based Science Education lo studio qui proposto illustra il processo di costruzione e validazione di un nuovo questionario (DIDLab) finalizzato alla misura del grado di conoscenza dell'approccio Inquiry e dello stile di conduzione nella didattica laboratoriale dei docenti in formazione quali metodologie da utilizzare in sinergia.

La validazione del questionario DIDLab è stata altresì finalizzata alla valutazione delle performance dello strumento per migliorare lo stile di conduzione della didattica laboratoriale utile a facilitare l'acquisizione di una competenza.

\section{Quadro teorico}

Gli studenti non sono delle menti su cui 'incidere' concetti, ma riescono a offrire intuitivamente delle spiegazioni circa il funzionamento della realtà che li circonda. Per tale ragione è opportuno spingere verso una formazione in cui gli studenti siano competenti e capaci di interagire con la conoscenza in modo 
responsabile, consapevole e autonomo (Budnyk, 2019). Sin dalla scuola dell'infanzia è necessario adottare uno stile di insegnamento diverso dalla tradizionale didattica frontale e che vada verso una didattica di tipo laboratoriale e di ricerca (Biondi, Borri \& Tosi, 2016). Gli studenti devono essere sollecitati a esperienze pratiche: la ricerca sul campo, lo studio delle fonti 'dirette' assumono un valore fondamentale in quanto lo studente impara come porsi nei confronti della vita al di fuori della scuola.

Tutto questo si coniuga perfettamente con le possibilità che può offrire un laboratorio scientifico Inquiry-Based. Negli studi in didattica è noto che quando si parla di metodologie, quella laboratoriale e quella investigativa rappresentano sovente due modi diversi di organizzare l'insegnamento (Gallina, Irato \& Santovito, 2019).

Le caratteristiche essenziali di un'attività laboratoriale secondo Munari (Fabbri \& Munari, 1994) sono: la manipolazione concreta; la richiesta di operazioni cruciali; la ricerca di una o più soluzioni; la stimolazione dello 'spiazzamento cognitivo'; il coinvolgimento delle conoscenze pregresse. Alla luce di quanto descritto è possibile coniugare le caratteristiche della didattica laboratoriale con il metodo investigativo; di fatti il laboratorio inquiry presuppone un livello di ricerca più elevato rispetto al laboratorio classico, in cui potrebbe esserci più attenzione all' aspetto operativo rispetto a quello metacognitivo (Gunn, 2018; Mariani, 2008). È noto che le attività laboratoriali basate sul modello Inquiry portano a risultati migliori per gli studenti rispetto ai corsi di laboratorio tradizionali (Beck, Butler \& Burke da Silva, 2014; Weaver, Russell \& Wink, 2008).

I due metodi sono, quindi, perfettamente complementari: condurre un laboratorio Inquiry vuol dire mettere in atto una didattica operativa, caratterizzata da un ciclo di indagine tipico di un metodo investigativo (Tavares, 2015).

In letteratura una definizione abbastanza comune di Inquiry è quella data da Linn, Davis e Bell (2004) che lo definiscono un processo intenzionale di diagnosi di problemi, analisi critica di situazioni, distinzione tra varie possibili alternative, pianificazione di attività di studio ed esplorazione, costruzione di congetture, ricerca di informazioni, costruzione di modelli, confronto in un contesto fra pari ed elaborazione di argomentazioni coerenti.

L'IBSE è una strategia educativa in cui gli studenti seguono metodi e pratiche simili a quelle dei ricercatori al fine di costruire la conoscenza; esso può essere definito come un processo di scoperta dove ogni allievo formula ipotesi, conduce esperimenti e fa osservazioni (Zacharias et al., 2015).

L'Inquiry based laboratory approach viene adottato per la prima volta in America, intorno agli anni '70 del secolo scorso, da Rosalind Driver. Dalla sua sperimentazione l'interesse per tale pratica didattica laboratoriale è cresciuto poiché nel corso dei decenni e grazie alle ricerche, si è ampiamente dimostrata 
la sua efficacia nel processo di insegnamento-apprendimento. L'impianto Inquiry gioca un ruolo fondamentale nello sviluppo e nel potenziamento di competenze negli studenti perché richiede l'orchestrazione e la contestualizzazione di risorse interne ed esterne della persona in situazioni reali, ricorrendo a metodi di insegnamento più strutturati e organizzati (Qablan et al., 2008).

Gli studi e le ricerche sul modello Inquiry sottolineano che l'adozione del modello deve avvenire attraverso un percorso progressivo che parte dalle consuete attività didattiche in aula, in cui l'insegnante è il fulcro dell'attenzione, e gradualmente lo studente diviene elemento cardine del processo formativo. Banchi \& Bell (2008) individuano quattro livelli di Inquiry: Confermativo, Strutturato, Guidato e Aperto, in quest'ordine si passa da una forte partecipazione dell'insegnante e scarsa dello studente ad una partecipazione attiva dell'allievo.

Nell'Inquiry confermativo, l'insegnante, introdotto un nuovo concetto lascia gli studenti sperimentare quella nuova conoscenza attraverso un'attività di laboratorio dove gli studenti seguiranno la guida dell'insegnante per condurre l'attività sperimentale e traggono le loro conclusioni a seguito dell'analisi dei dati raccolti.

Nell'Inquiry strutturato si chiede agli studenti di anticipare lo studio dei concetti che saranno spiegati in seguito, e di raccogliere e analizzare i dati interiorizzando i concetti.

Nell'Inquiry guidato gli studenti non solo si documentano sui concetti teorici ma pianificano attività pratiche guidate per fare esperienze di quei concetti.

Nell'Inquiry aperto gli studenti sono del tutto autonomi e il docente avrà il solo ruolo di valutare la correttezza delle loro costatazioni e apprendimenti. Tale tipologia di Inquiry è perseguibile solo se gli studenti hanno avuto ampia esperienza dei primi tre livelli.

I 4 livelli di Inquiry rappresentano le diverse fasi di un processo continuo in cui i docenti permettono agli studenti di progredire in autonomia e nella ricerca partendo dai livelli più bassi di partecipazione attiva a quelli più alti, fino ad ottenere livelli elevati di competenze che gli permetteranno di svolgere ricerche aperte.

Il modello Inquiry laboratory prevede l'utilizzo di strumenti e modelli didattici specifici che i docenti dovrebbero utilizzare per ipotizzare i percorsi laboratoriali (Abraham, 2005; Siddiqui et al., 2013).

Dall'unione del metodo investigativo con la didattica laboratoriale nasce l'Inquiry based laboratory, caratterizzato da un processo di indagine tipico del metodo dell'Inquiry-Based Science Education (IBSE). L'Inquiry laboratory segue il processo ciclico IBSE: domandare (Ask), ricercare (Investigate), crearecostruire (Create), discutere (Discuss), riflettere (Reflect). 
L'approccio Inquiry-Based pone il discente verso un atteggiamento di ricerca che designa il cambio di prospettiva dell'apprendimento. In un insegnamento tradizionale l'apprendimento è il fine ultimo dell'azione didattica, in un Laboratorio Inquiry l'apprendimento rappresenta il mezzo attraverso il quale lo studente mette in atto le conoscenze possedute. Con il metodo IBSE appare evidente il ruolo centrale dello studente ma risulta essere essenziale anche il ruolo del docente: egli assume un ruolo di guida, in grado di sviluppare negli studenti le competenze attraverso lavori di gruppo che inducono alla discussione e al dialogo, allo scambio di informazioni e alla sperimentazione.

\section{Il processo di validazione del questionario DIDLab}

\subsection{Soggetti}

Durante il IV ciclo del corso di specializzazione per le attività del sostegno didattico della scuola dell'infanzia e della scuola primaria dell'Università degli Studi di Palermo, i laboratori previsti sono stati progettati sulla base dell'approccio IBSE. I destinatari dell'intervento sperimentale sono i 523 corsisti iscritti sui quali si è voluto misurare il grado di conoscenza dell'approccio Inquiry-Based Science Education e gli stili di conduzione da loro applicati durante l'espletamento di un laboratorio. Del campione in oggetto il 56\% (292) svolge servizio (Figura 1) nella scuola primaria, il 20\% (104) nella scuola dell'infanzia, 2,3\% (12) nelle scuole secondarie di primo e secondo grado, circa il 20\% (106) non insegna e il restante 1,7\% (9) si divide tra scuola paritaria, istituzione educativa e centri d'accoglienza per minori.

Il campione si suddivide sotto l'aspetto degli anni di servizio (figura 2), all'interno di questi range: il 27\% (140) tra i 2 e i 5 anni, il 18\% (94) tra i 10 e i 20 anni, il 16\% (85) tra i 5 e i 10 anni, il 14\% (73) tra 1 e 2 anni, il 10\% (53) meno di un anno e il rimanente $15 \%$ (78) non ha ricoperto alcun servizio.

Relativamente agli incarichi (Figura 3), il 35\% (187) afferma di aver avuto incarichi sul sostegno, mentre il $27 \%$ (142) sul posto comune e la restante parte ha ricoperto altri incarichi (personale educativo, operatore specializzato, dirigente scolastico, ecc.).

Il campione nel grado di specializzazione (Figura 4) si suddivide nel 25\% circa su infanzia e il quasi $75 \%$ su primaria. 


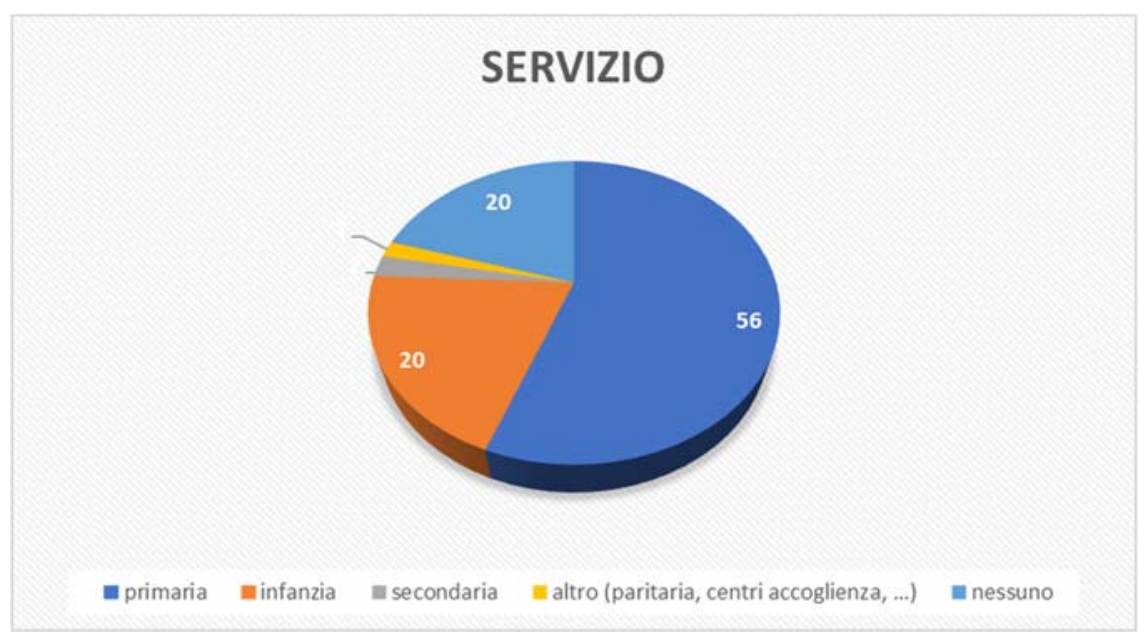

Figura 1- Suddivisione del campione: servizio

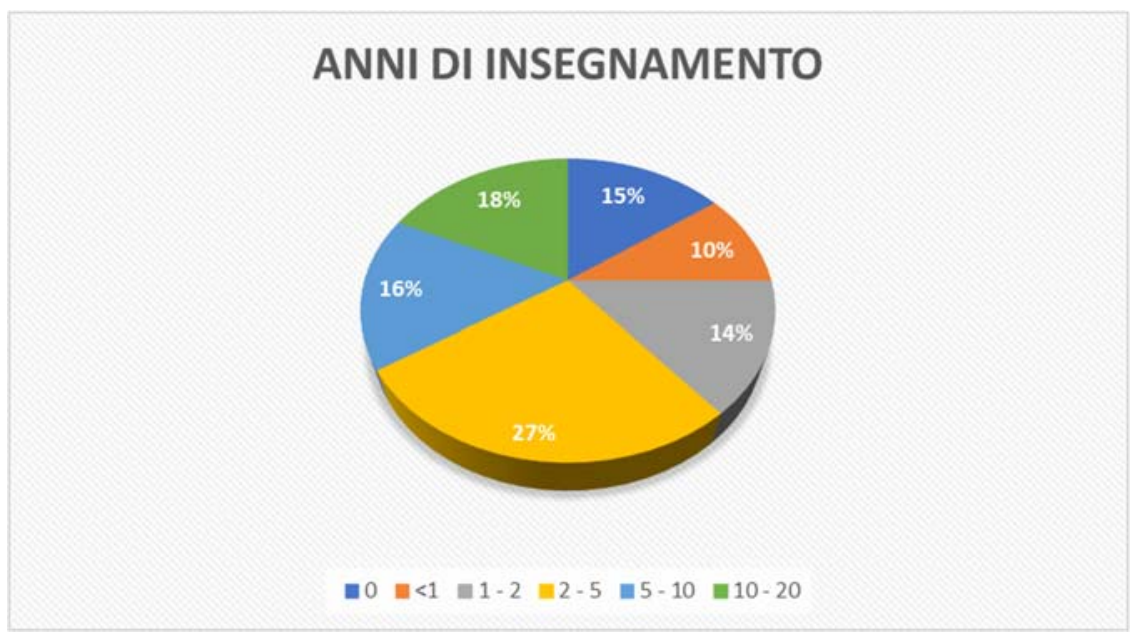

Figura 2- Suddivisione del campione: anni di servizio 


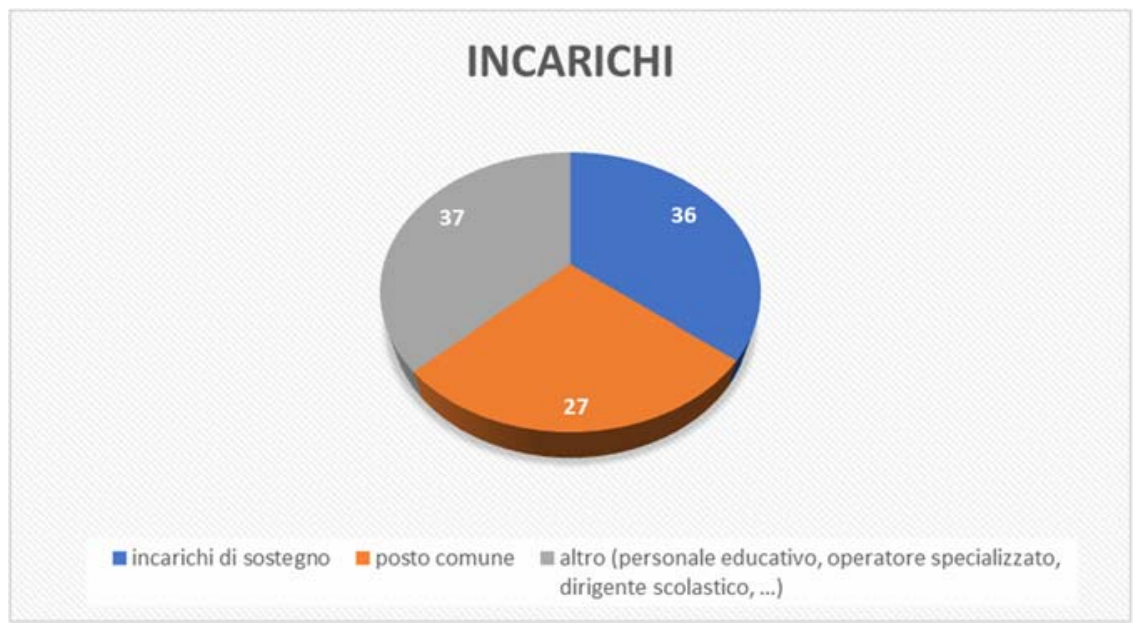

Figura 3- Suddivisione del campione: incarichi

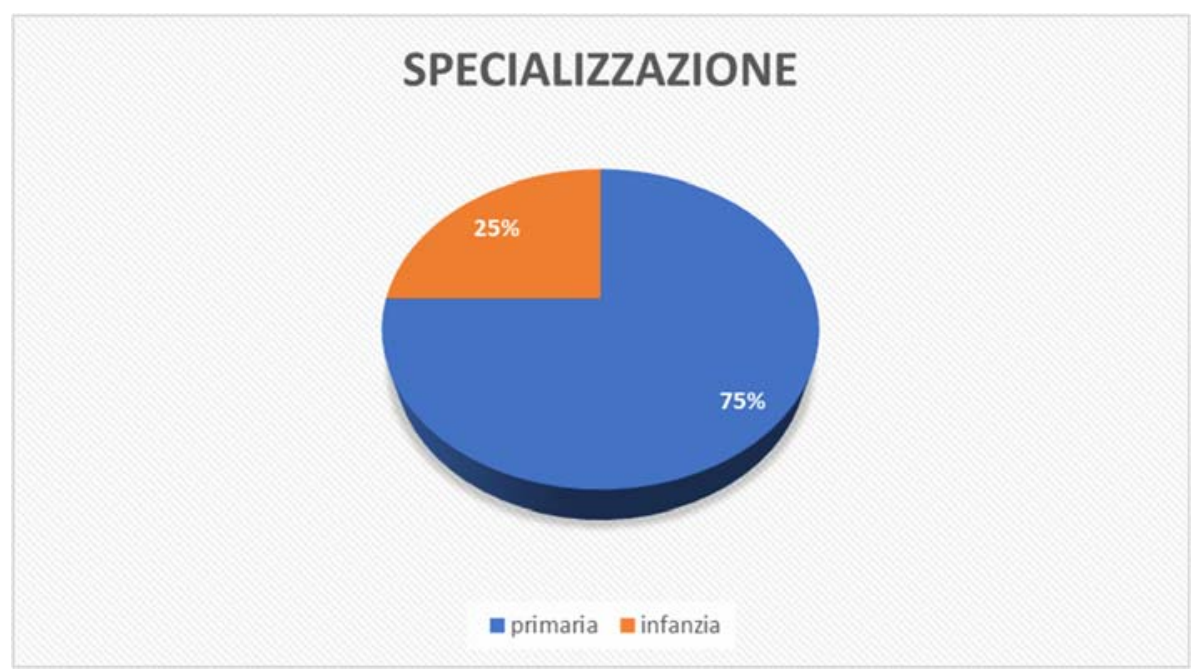

Figura 4- Suddivisione del campione: scuola di specializzazione

\subsection{Il Questionario DIDLab}

Il test di misurazione dal titolo DIDLab "La didattica Laboratoriale: apprendimenti e stili di conduzione con l'approccio IBSE", è stato da noi elaborato ed è costituito da 42 item. 
Scopo della validazione è quello di ottenere uno strumento in grado di misurare il livello di conoscenza e di utilizzo dell'approccio Inquiry-Based nella didattica laboratoriale, investigando su cinque macroaree:

1) generale: conoscenza dell'approccio Inquiry Based Laboratory;

2) docente-conduttore: funzioni e compiti del docente durante un laboratorio IBSE;

3) studente: funzioni e compiti dello studente durante un laboratorio IBSE;

4) valutazione didattica: strumenti di valutazione utilizzati in un laboratorio Inquiry-Based;

5) stili di conduzione del laboratorio: valutazione del livello di Inquiry dei laboratori secondo la suddivisione di Banchi \& Bell (2008).

Al campione di riferimento è stato chiesto di esprimere il proprio grado di accordo o disaccordo alle affermazioni proposte secondo una scala Likert a 5 punti (da 1 "Per niente d'accordo" a 5 "Del tutto d'accordo"). La scala, infatti, ha il vantaggio di non lavorare su due situazioni bipolari, ma permette al soggetto di collocarsi ad un livello intermedio rispetto alle due situazioni opposte.

Lo strumento è stato somministrato all'intero campione di riferimento prima (luglio 2019) e dopo (aprile 2020) le sessioni laboratoriali.

Il percorso di validazione dello strumento si è articolato in diverse fasi:

Fase 1. Il primo passo della ricerca è stato finalizzato alla produzione di una gamma di Item (83) ritenuti rappresentativi dell'approccio Inquiry-Based Science Education. Tali Item sono stati valutati da due ricercatori. Soltanto 64 item sono stati approvati con pieno giudizio e inclusi nel prototipo dello strumento.

Fase 2. La versione ottenuta nella fase precedente è stata somministrata ad un campione di 157 studenti dei corsi di Laurea Magistrale in Scienze Pedagogiche e di Scienze della formazione Continua dell'Università degli Studi di Palermo per verificare la comprensione degli item. Sono stati eliminati gli item di più difficile comprensione sulla base del thinking aloud, ovvero una procedura nella quale al soggetto è stato chiesto di descrivere ciò che viene compreso della lettura degli item proposti.

Fase 3. La seconda versione dello strumento, composta da 53 item è stata somministrata a un campione di 161 studenti del corso di Laurea Magistrale in Scienze della Formazione Primaria dell'Ateneo palermitano. Sulla base dei risultati è stata scelta una soluzione a cinque fattori al fine di ridurre la varianza e sono stati selezionati gli item con le caratteristiche psicometriche più attendibili, ottenendo la versione definitiva dello strumento.

Fase 4. La terza versione dello strumento, che constava di 42 item è stata sottoposta dal campione oggetto di indagine costituito da 523 corsisti del corso di specializzazione per le attività di sostegno didattico. I dati ottenuti sono stati 
elaborati a mezzo di analisi fattoriale e sono stati verificate la correttezza e l'attendibilità degli item.

Fase 5. Un'ulteriore verifica dell'attendibilità è stata effettuata per mezzo dell'analisi esplorativa e confermativa dei fattori.

Fase 6. Per individuare eventuali differenze ascrivibili al grado di istruzione e agli anni di servizio è stata effettuata un'analisi della varianza sui dati ottenuti dalla somministrazione del questionario ai 523 corsisti, utilizzando come variabili indipendenti il grado scolastico in cui si presta servizio e gli anni di insegnamento come variabili dipendenti il punteggio ottenuto nei fattori preponderanti ottenuti dall'analisi fattoriale.

\subsection{Modalità di Somministrazione}

La somministrazione dei questionari è stata svolta tramite la piattaforma Google Moduli al fine di raggiungere in maniera immediata e capillare tutti i soggetti coinvolti nelle varie sessioni di somministrazione del percorso di validazione dello strumento.

La somministrazione del prototipo dello strumento consistente in 64 item è stata effettuata nel mese di maggio 2019; la seconda versione dello strumento, di 53 item, è stata somministrata a giugno 2019; infine, la somministrazione della versione definitiva ai 523 corsisti, è avvenuta in occasione dell'avvio del corso di specializzazione (luglio 2019) e si è ripetuta al termine del corso (aprile 2020).

L'utilizzo delle piattaforme digitali per la somministrazione dei questionari è risultata lungimirante data l'esplosione dell'emergenza sanitaria vissuta nel marzo 2020.

\section{Analisi dei dati}

I dati ottenuti utili per la misurazione del grado di conoscenza e utilizzo dell'approccio Inquiry-Based Science Education nella didattica laboratoriale sono stati analizzati attraverso l'ausilio del software di analisi statistica IBM SPSS v23 al fine di valutare l'affidabilità dei fattori, la presenza di correlazioni e le differenze basate sul grado di istruzione e anni di servizio.

L'analisi di affidabilità è stata effettuata calcolando l'Alfa di Cronbach e calcolando la correlazione tra i singoli item e la scala totale. Per l'analisi fattoriale è stato utilizzato il metodo di estrazione dei fattori principali (AFP) e sono stati analizzati la correttezza e l'attendibilità dei fattori principali. Successivamente sui fattori individuati è stata condotta un'analisi esplorativa. Infine, è 
stata condotta un'analisi della Varianza Multivariata (MANOVA) facendo emergere delle differenze dovute a anni di servizio e grado di istruzione.

\section{I risultati}

\subsection{Analisi dell’Affidabilità}

L'area "Generale" presenta un Alfa di Cronbach pari a 0,669 ma non tutti gli item mostrano una correlazione item-scala pari a 0,30 . Si è proceduto, dunque, all'eliminazione dell'item con il valore di correlazione item-scala più basso (item 1), pari a 0,041 . E successivamente all'item 3 con un valore di correlazione pari a 0,145 . L'eliminazione degli item in questione ha comportato un aumento dell'alpha di Cronbach pari a 0,73.

L'area "Docente-Conduttore" presenta un'alpha di Cronbach di molto superiore alla sufficienza accettabile 0,891 , nonostante ciò si è proceduto all'eliminazione degli item $11,12,15$ e 18, con un valore di correlazione item-scala pari rispettivamente a $0,219,0,071,0,122$ e 0,051 . L'eliminazione degli item ha comportato un significativo aumento del valore dell'alpha di Cronbach pari a 0,920 .

L'area "Studente" presenta un'alpha di Cronbach convenzionalmente accettabile, pari a 0,625 . Dall'analisi di affidabilità condotta vengono eliminati gli item $35,37,40$ e 41 perché il valore di correlazione item-scala è inferiore a 0,30 . Tale eliminazione aumenta il valore dell'alpha di Cronbach uguale a 0,770 .

L'area "Valutazione didattica" presenta un valore poco superiore al limite accettato pari a 0,561 e solo due item (44 e 47) presentano una correlazione item-scala inferiore al minimo accettabile. L'eliminazione di suddetti item provoca un aumento dell'alpha di Cronbach pari a 0,686.

Infine, l'area "Stili di conduzione" registra un valore di alpha di Cronbach pari a 0,806 . Vengono eliminati gli item 49, 50, 54, 62 e 64 .

\subsection{Analisi Fattoriale Esplorativa}

L'analisi fattoriale esplorativa, in cui sono stati analizzati tutti gli item delle cinque aree individuate, ha evidenziato la presenza di quattro fattori come si evince dallo screen-test (Figura 5) in cui si prendono in considerazione i fattori con valore superiore all'unità. Si è scelto di estrarre due fattori che spiegano il $36 \%$ di varianza totale. 


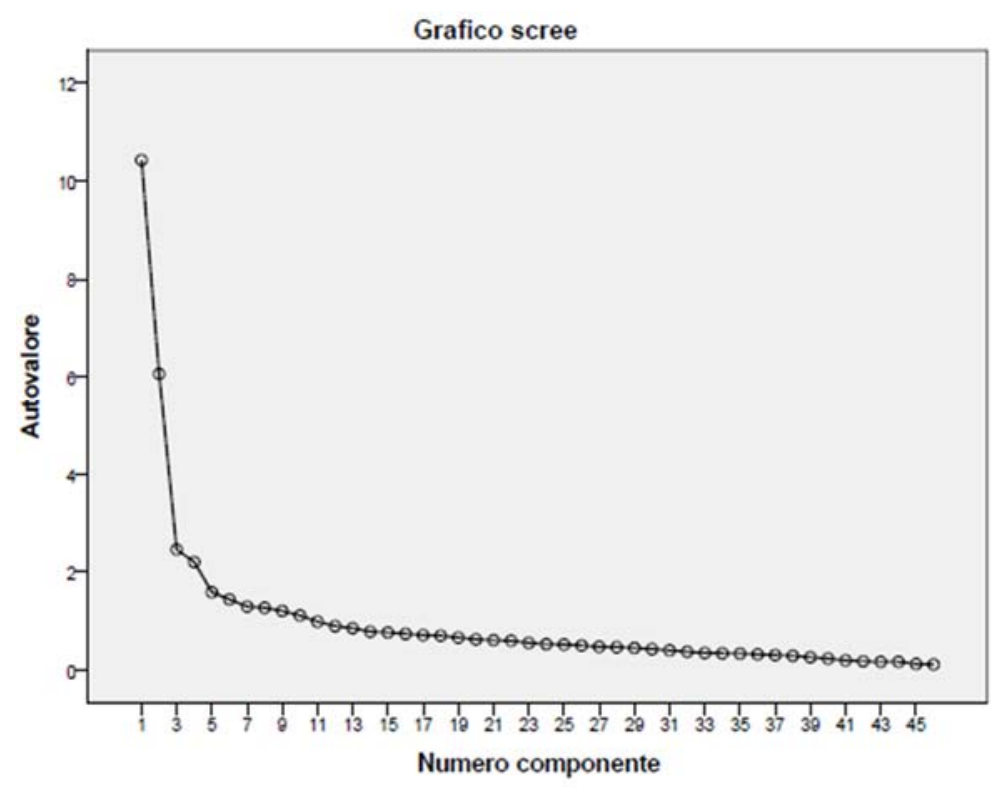

Figura 5- Grafico Screen

Il primo fattore spiega il 23\% della varianza totale e i cui item appartengono all' area investigativa sugli stili di conduzione della didattica laboratoriale. Tale fattore, comprendente di 23 item $(13,14,16,17,19,20,21,22,23,24,25,26$, $27,28,29,30,31,42,43,45,46,55$ e 58) comprendenti le aree Docente-conduttore, Studente e Stili di conduzione nella loro totalità; tale fattore registra un valore dell'alpha di Cronbach pari a 0,931 . Tale fattore verrà nominato Stile di conduzione nella didattica laboratoriale ed è costituito dagli item che richiedono le tecniche e le metodologie utilizzate durante la didattica laboratoriale, $\mathrm{i}$ compiti del docente-conduttore e dello studente e l'individuazione dei punti di forza e di criticità nell'utilizzo della didattica laboratoriale rispetto ai metodi tradizionali.

Il secondo fattore spiega la varianza totale al $13 \%$ ed è sovrapponibile all'area Generale. Abbiamo nominato questo fattore Conoscenza dell'approccio IBSE in quanto coincide con la conoscenza e l'utilizzo dell'approccio Inquiry-Based Science Education e gli stili di conduzione propri di tale metodologia.

\subsection{Analisi della Varianza Multivariata}

Al fine di esplorare differenze ascrivili agli anni di servizio e al grado di istruzione dove si presta insegnamento è stata condotta un MANOVA 2 X 2 (Stile di conduzione della didattica laboratoriale e Conoscenza dell'approccio 
IBSE vs Anni di servizi e Grado di istruzione) al fine di mostrare una differenza tra le diverse dimensioni (Tabella 1). Nella seguente analisi sono state considerate come variabili indipendenti gli anni di servizio e il grado di istruzione e come variabili dipendenti i punteggi ottenuti nei due fattori emersi dall'analisi fattoriale. Per quanto concerne il grado di istruzione questo ha visto coinvolti i quattro ordini scolastici a cui è rivolto il corso di specializzazione $(0=$ non insegno; 1 = scuola dell'infanzia; 2 = scuola primaria; 3 = scuola secondaria di primo grado; 4 = scuola secondaria di secondo grado). Per quanto riguarda gli anni di servizio sono stati suddivisi in 5 fasce $(0=$ non insegno; $1=$ da meno di 1 anno; 2 = 1 anno a 2 anni; 3 = da 2 a 5 anni; $4=$ da 5 a 10 anni; $5=$ da 10 a 20 anni).

Per rilevare una possibile relazione tra gli anni di servizio e il grado di servizio e le conoscenze e l'applicazione dell'approccio Inquiry-Based Science Education è stata effettuata una correlazione tra le medie dei punteggi ottenuti e le due variabili individuate. Una volta aver verificato che le matrici di varianza osservate delle variabili dipendenti siamo uguali tra i gruppi (Box's $M=$ $385,939 ; F=1,379 ; p=, 000)$ il test multivariato ha mostrato una correlazione significativa per entrambi i fattori.

Nello specifico, i punteggi ottenuti dal questionario hanno evidenziato valori positivi di chi insegna da almeno 5 anni nella Scuola dell'infanzia e Primaria. Una correlazione negativa viene invece indicata rispetto a chi insegna da più di un anno e a 2 anni negli ordini di scuola dell'infanzia, primaria e secondaria di primo grado: viene osservata una diminuzione dei punteggi nella Conoscenza dell'approccio Inquiry.

In riferimento alla scala Grado di servizio, chi insegna nella scuola Secondaria di Secondo grado risulta conoscere meno l'approccio Inquiry-Based Science Education, seppur registri valori medio-alti nell'applicazione e utilizzo. In particolare, chi insegna da almeno 10 anni registra valori più alti nella dimensione applicazione di tale approccio nella didattica laboratoriale. 


\begin{tabular}{|c|c|c|c|c|c|c|c|c|c|c|}
\hline 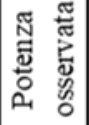 & $\stackrel{m}{\stackrel{*}{ } ~}$ & Iี & క. & 은 & 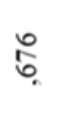 & $\hat{n}$ & ડે. & ڤ̆ & ڤ & $\hat{a}$ \\
\hline 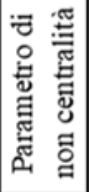 & $\begin{array}{l}\stackrel{2}{\circ} \\
\stackrel{\circ}{0} \\
\dot{\Xi}\end{array}$ & 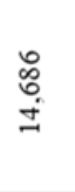 & $\begin{array}{l}\hat{0} \\
\stackrel{0}{0}\end{array}$ & 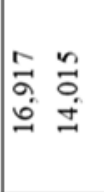 & $\begin{array}{l}\text { aे } \\
\text { ठِ }\end{array}$ & $\begin{array}{l}\stackrel{N}{气} \\
=\end{array}$ & $\mid \begin{array}{l}n \\
\tilde{n} \\
\hat{n} \\
\tilde{n}\end{array}$ & 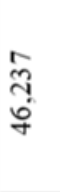 & जे & $\begin{array}{l}\text { \&े } \\
\text { के }\end{array}$ \\
\hline 离 & †े & $\stackrel{\infty}{a}$ & s. & $\begin{array}{l}\infty \\
0 \\
0 \\
0\end{array}$ & $\begin{array}{l}\infty \\
0 \\
0\end{array}$ & 웅. & $\stackrel{n}{n}$ & $\stackrel{q}{q}$ & 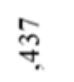 & ธิ \\
\hline $\begin{array}{l}\frac{2}{0} \\
\vdots \\
5 \\
0\end{array}$ & 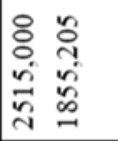 & 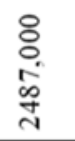 & $\begin{array}{l}\text { ¿े } \\
\text { ô. } \\
\text { in }\end{array}$ & 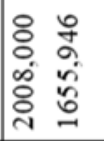 & 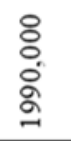 & $\begin{array}{l}\text { ¿े } \\
\text { ì } \\
\text { ì }\end{array}$ & 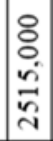 & 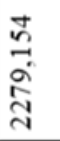 & 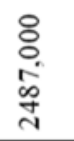 & $\begin{array}{l}\text { ¿े } \\
\text { î̀ } \\
\text { in }\end{array}$ \\
\hline 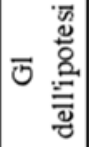 & $\begin{array}{l}\circ \\
\stackrel{0}{\circ} \\
\dot{y} \\
\dot{y}\end{array}$ & $\begin{array}{l}\text { ¿ } \\
\text { ¿̀ं }\end{array}$ & ¿ & 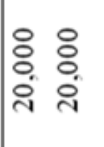 & : & : & 吕 & ¿̊. & : & $\begin{array}{l}\circ \\
\stackrel{\circ}{\circ}\end{array}$ \\
\hline 山 & $\begin{array}{l}\infty \\
\infty \\
\infty \\
n \\
n \\
n\end{array}$ & $\stackrel{\infty}{n}$ & $\stackrel{n}{0}$ & 占 & 占 & $\begin{array}{l}\text { : } \\
\text { in } \\
\text { co }\end{array}$ & $\overrightarrow{\vec{\sigma}}$ & $\stackrel{n}{-}$ & $\stackrel{\circ}{\circ}$ & $\begin{array}{l}\text { ¿े } \\
\text { i } \\
\text { i }\end{array}$ \\
\hline$\frac{\tilde{y}}{\frac{0}{\pi}}$ & ఫิ & ర్లి & కి. & ๓ీ & ô. & న్ర & : & ธั & $\stackrel{\overbrace{}}{\varrho}$ & 웅 \\
\hline 苞 & 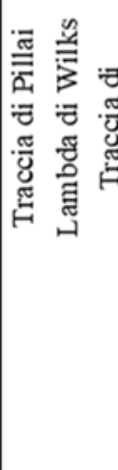 & 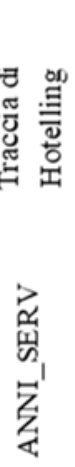 & 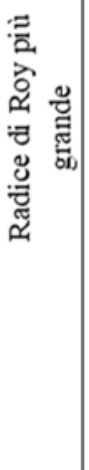 & 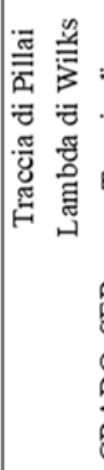 & 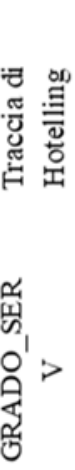 & 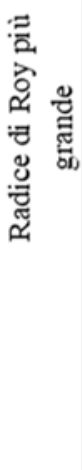 & 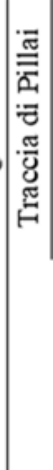 & 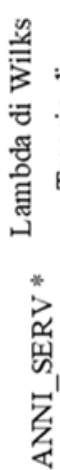 & 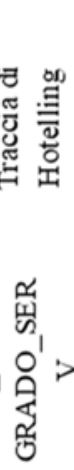 & 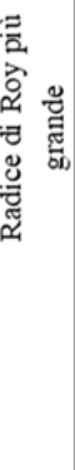 \\
\hline
\end{tabular}

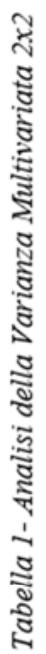




\section{Conclusioni}

Il questionario oggetto del presente lavoro è uno strumento utile a misurare la conoscenza dell'approccio investigativo e il suo utilizzo nella didattica laboratoriale. Tale strumento è finalizzato a favorire percorsi di confronto e di autovalutazione e in grado di promuovere, nel docente in formazione, una maggiore consapevolezza del proprio stile di conduzione e del processo di insegnamento in generale. Dall'analisi sono emersi risultati molto soddisfacenti per quanto riguarda l'attendibilità e la validità del questionario costruito. Inoltre è possibile ritenere che questo possa essere utilizzato come strumento atto a misurare la conoscenza dell'approccio Inquiry-Based Science Education e l'utilizzo di tale approccio in tutti gli ordini scolastici.

L'analisi dell'affidabilità condotta sui dati ottenuti da una prima somministrazione del questionario ha permesso di raccogliere alcuni item sotto le cinque macro-aree individuate (Generale, Docente-conduttore, Studenti, Valutazione e Stili di conduzione) e di eliminare quelli che presentava una scarsa correlazione item-scala.

Dall'analisi fattoriale condotta sono stati estrapolati i due fattori più rappresentativi della varianza totale rispetto ai quattro emersi, i quali raggruppano le cinque aree individuate: il primo fattore, denominato Stili di conduzione nella didattica laboratoriale, indaga sullo stile di conduzione utilizzato riprendendo i compiti del "docente-conduttore" e dello studente rispetto al metodo investigativo; il secondo fattore individuato, denominato Conoscenza dell'approccio Inquiry-Based Science Education, studia invece le conoscenze possedute sull'approccio dai docenti in formazione.

Infine, è stata condotta un MANOVA 2 X 2 per esplorare differenze ascrivili agli anni di servizio e al grado di istruzione dove si presta insegnamento. Tale indagine ha rilevato una correlazione tra la conoscenza e l'applicazione dell'approccio IBSE alla didattica laboratoriale tanto maggiore quanto più sono gli anni di servizio e all'interno degli ordini di scuola primaria e secondaria di primo grado.

La conoscenza e l'utilizzo dell'approccio Inquiry-Based Science Education, applicato alla didattica laboratoriale, risulta essere di fondamentale importanza, non solo per poter rinnovare il sistema di istruzione italiano passando da una didattica meramente nozionistica e radicata sul libro di testo a una didattica laboratoriale volta a favorire la trasferibilità di una competenza, ma offre ai docenti l'opportunità di poter sperimentare un nuovo metodo di insegnamentoapprendimento che rende il soggetto consapevole delle risorse di cui dispone.

I dati ottenuti dalla presente ricerca ricalcano quanto finora dichiarato in altri studi, ovvero che l'approccio investigativo nella didattica laboratoriale non viene sempre utilizzato per mancanza di conoscenza e sperimentazione. 


\section{Riferimenti bibliografici}

Ausubel, D.P (1963). The Psychology of Meaningful Learning. Newyork: Grune and Stratton

Ausubel, D. P. (2004). Educazione e processi cognitivi. Guida psicologica per gli insegnanti (Vol. 25). Milano: FrancoAngeli.

Abraham, M. R. (2005). Inquiry and the learning cycle approach. Chemists' guide to effective teaching, 1, 41-52.

Banchi, H., \& Bell, R. (2008). The many levels of inquiry. Science and children, 46(2), 26.

Beck, C., Butler, A., \& Burke da Silva, K. (2014). Promoting inquiry-based teaching in laboratory courses: are we meeting the grade?. CBE - Life Sciences Education, 13(3), 444-452.

Biondi, G., Borri, S., \& Tosi, L. (Eds.) (2016). Dall'aula all'ambiente di apprendimento (Vol. 4). Altralinea Edizioni.

Budnyk, O. (2019). Innovative Competence of a Teacher: Best European Practices, Journal of Vasyl Stefanyk Precarpathian National University, 6(1), 76-89.

Cappuccio, G., \& Maniscalco, L. (2020). Physical and Inclusive Education. Un percorso di ricerca Inquiry based con i futuri docenti di sostegno. Giornale Italiano di Educazione alla Salute, Sport e Didattica Inclusiva, 4(4 sup).

Gallina, S., Irato, P., \& Santovito, G. (2019). Inquiry into animal tracks: an experimental application of IBSE-inquiry based science education-approach in the ecological field in primary school. INTED2019 Proceedings.

Gunn, J. (2018). The Art of Inquiry in STEAM Education. https://education.cuportland.edu/blog/classroom-resources/steam-inquiry-based-learning/.

Fabbri, D., \& Munari, A. (1994). I laboratori di epistemologia operativa.

Fassinato, C. et al. (2018). An innovative approach to deal with biodiversity at school with its investigation in different environments. In Gómez Chova, L., López, Martínez, A., Candel Torres, I. (Eds). EDULEARN18 Proceedings of the 9th International Conference on Education and New Learning Technologies, Barcelona, Spain, 2-4 July, Valencia: IATED, pp. 2671-2678.

Linn, M. C., Davis, E. A., Bell, P. (2004). Internet Environments for Science Education. Mahwah, New Jersey: Lawrence Erlbaum Associates.

Mariani, A. (2008). La decostruzione in pedagogia: una frontiera teorico-educativa della postmodernità. Armando Editore.

Qablan, A., Al-Ruz, J. A., Theodora, D., \& Al-Momani, I. (2008). "I Know It's So Good, But I Prefer Not To Use It" An Interpretive Investigation of Jordanian Preservice Elementary Teachers' Perspectives about Learning Biology Through Inquiry. International Journal of Teaching and Learning in Higher Education, 20(3), 394-404.

Righetti, M. (2007). Organizzazione e progettazione formativa (Vol. 83). FrancoAngeli.

Siddiqui, S., Zadnik, M., Shapter, J., \& Schmidt, L. (2013). An inquiry-based approach to laboratory experiences: Investigating students' ways of active learning. International Journal of Innovation in Science and Mathematics Education, 21(5). 
Tavares, A.C., Silva, S. \& Bettencourt, T. (2015). Vantaggi di educazione scientifica all'aperto attraverso la metodologia IBSE. Innovazione nell'istruzione superiore. Insegnamento e apprendimento, 4, 151-169.

Uzezi Gladys, J. \& Zainab, S. (2017). Effectiveness of Guided-Inquiry Laboratory Experiments on Senior Secondary Schools Students Academic Achievement in Volumetric Analysis. American Journal of Educational Research, 5(7), pp.717-724.

Weaver, G. C., Russell, C. B., \& Wink, D. J. (2008). Inquiry-based and research-based laboratory pedagogies in undergraduate science. Nature chemical biology, 4(10), 577-580.

Zacharias, Z.C., Manoli, C., Xenofontos, N., de Jong, T., Pedaste, M., van Riesen, S., Kamp, E., Mäeots, M., Siiman, L., Tsourlidaki, E. (2015). Identifying potential types of guidance for supporting student inquiry when using virtual and remote labs in science: a literature review. Educational technology research and development, 63(2), 257-302. 